\section{Spermien aus Hoden oder Ejakulat?}

Wenn Spermien aus dem Ejakulat für eine intrazytoplasmatische Spermieninjektion (ICSI) nicht verfügbar sind, kann die Entnahme von Spermien aus den Testes möglicherweise eine Option sein. Hinweise dafür liefern die Ergebnisse einer retrospektiven Studie mit Männern, bei denen eine Kryptozoospermie diagnostiziert worden war. Für die chinesische Studie wurden die Befunde von fast 300 Männern ausgewertet. Wie die Ärzte berichten, waren die Erfolgsraten bei Implantation, Schwangerschaft und Geburt mit aus den Hoden statt aus dem Ejakulat gewonnenen Spermatozoen signifikant höher.

\section{Adipositas verbessert Überleben bei RCC}

Der Einfluss des Body-Mass-Index (BMI) bei Patienten mit Nierenzellkarzinom (renal cell carcinoma, RCC) steht schon seit Jahren im Fokus medizinischer Studien. Eine Studie aus den USA bestätigt nun den Überlebensvorteil von RCC-Patienten mit einem BMI über $30 \mathrm{~kg} / \mathrm{m}^{2}$. Im Rahmen dieser Studie wurden Daten von 845 RCC-Patienten aus 14 Ländern erhoben. Es zeigte sich, dass sich sowohl das krankheitsfreie Überleben als auch das Gesamtüberleben mit zunehmendem BMI-Wert signifikant verbesserte. $\mathrm{Ob}$ die paradoxe Korrelation tatsächlich auf den BMI selbst oder doch auf die individuelle Konstitution zurückzuführen ist, bleibt unklar.

Cancer Epidemiol Biomarkers Prev. 2016; 25(9):1326

\section{Gleichberechtigung beim Impfen!}

Gebärmutterhalskrebs, Analkarzinome, Kopf-Hals-Tumoren: Die Liste der von humanen Papillomviren hervorgerufenen Krankheitsbilder ist lang und schwerwiegend. Vor diesem Hintergrund empfehlen Experten, nicht nur Mädchen, sondern auch Jungen und Männer konsequent gegen HPV zu impfen. Wie erfolgreich solche Maßnahmen sind, zeigen z.B. Daten aus dem Ausland. Lesen Sie mehr zu diesem hochaktuellen Thema in unserem Schwerpunkt „Urologie“!

Claudia Daniels Redakteurin

\title{
Psoriasispatienten auf erektile Dysfunktion screenen?
}

Männer mit Psoriasis sollten chinesischen Forschern zufolge routinemäßig nach Erektionsstörungen befragt werden. Patienten, bei denen beides zusammentrifft, sind möglicherweise besondere Risikokandidaten für kardiovaskuläre Erkrankungen und Depressionen.

Die Studie aus dem chinesischen Guangzhou ist nicht groß, sie liefert aber deutliche Hinweise darauf, dass man eine erektile Dysfunktion (ED) bei Patienten mit Plaque-Psoriasis besonders ernst nehmen sollte. Die ED tritt bei Letzteren offenbar gehäuft auf und ist in besonderem Maße mit Risiken für das Herz-KreislaufSystem sowie mit Depressionen assoziiert.

Das Team um Dr. Suyun Ji vom Guangdong Provincial Dermatology Hospital hat eine Gruppe von 191 männlichen Psoriasispatienten aus fünf dermatologischen Zentren mit ebenso vielen hautgesunden Teilnehmern verglichen. In der Gruppe der Psoriatiker litten 52,9\% unter $\mathrm{ED}$, in der Vergleichsgruppe waren es 40,3\%. Dies entspricht einer adjustierten relativen Risikoerhöhung um den Faktor 1,97 in der Gruppe mit der Hauterkrankung. Die Männer in beiden Gruppen waren im Schnitt 45 Jahre alt. 38\% der Psoriatiker wiesen Hautveränderungen an den Genitalien auf.
Wie Ji und Kollegen berichten, trat die ED bei Psoriasispatienten besonders schwerwiegend in Erscheinung: 9,9\% wiesen eine mittelgradige, $5,8 \%$ eine schwere Form auf. In der Vergleichsgruppe waren es entsprechend $4,2 \%$ bzw. $1,6 \%$.

\section{Studien belegen Zusammenhang}

Im Vergleich zur Kontrollgruppe zeigte sich bei den Männern mit Psoriasis zudem häufiger Bluthochdruck (33,5\% gegenüber 19,9\%), Hyperlipidämie $(32,5 \%$ vs. $23,6 \%)$ und Diabetes mellitus (11,5\% vs. $5,2 \%$ ). Die Wahrscheinlichkeit einer ED war bei den Psoriatikern bei Vorliegen von Bluthochdruck um den Faktor 2,79 erhöht, bei Vorliegen einer Hyperlipidämie um den Faktor 2,47.

Der Zusammenhang zwischen der ED und einem erhöhten kardiovaskulären Risiko ist durch mehrere Studien belegt. Frühe atherosklerotische Veränderungen in den kleinen Beckengefäßen gelten heute als eine der Hauptursachen für die Erektionsstörung. Schätzungen gehen davon aus, dass vom Auftreten der ED an in der Regel zwei bis drei Jahre vergehen, bis es zu kardiovaskulären Symptomen kommt.

Ji S et al. International Journal of Impotence Research 2016; 28:96-100; doi: 10.1038/ijir.2016.6

\section{Cranberries schützen nicht vor HWI}

Bei Pflegeheimbewohnerinnen, die Cranberry-Kapseln schluckten, war ebenso häufig eine Bakteriurie plus Pyurie nachzuweisen wie in einer Vergleichsgruppe mit Placebokapseln. Teilnehmerinnen der randomisierten, kontrollier-

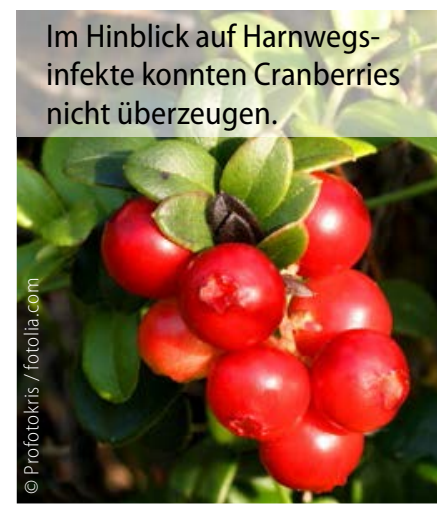
ten Studie waren 185 Frauen im mittleren Alter von 86 Jahren. 92 Frauen erhielten zweimal täglich eine Kapsel mit zusammen $72 \mathrm{mg}$ Proanthocyanidin, was etwa $600 \mathrm{ml}$ Cranberrysaft entspricht, die anderen 93 bekamen Placebokapseln. Alle zwei Monate wurde eine Urinprobe genommen: Eine Bakteriurie in Kombination mit einer Pyurie wurde in der Cranberrygruppe bei 25,5\% und in der Placebogruppe bei $29,5 \%$ festgestellt. Unter Berücksichtigung von fehlenden Daten und von vordefinierten Einflussfaktoren wurden die Anteile auf $29,1 \%$ und 29,0\% korrigiert, der Unterschied war nicht signifikant.

Juthani-Metha M et al. JAMA 2016, online 27. Oktober 